\title{
Surgery of Tetralogy of Fallot with Abnormal Coronary Arteries
}

\begin{abstract}
The Tetralogy of Fallot is the most common cyanotic congénital heart disease. The regular form combines from four anomalies: - A ventricular septal defect Pulmonary stenosis - Overriding aorta - A right ventricular hypertrophy. Irregular forms are all other forms where another heart defect is associated, among which are the anomalies of the coronary arteries found in 5-9\% of cases that require special care because of their complexity and anatomic varieties. The purpose of this study was to evaluate the particular therapeutic specificity of complete cure of Tetralogy of Fallot associated with a birth defect of the coronary arteries through a retrospective study of 9 cases collected in the cardiac surgery department at the International University Hospital Cheikh Zayed of Rabat.
\end{abstract}

Keywords: Tetralogy of Fallot, Congenital heart disease, Surgical repare

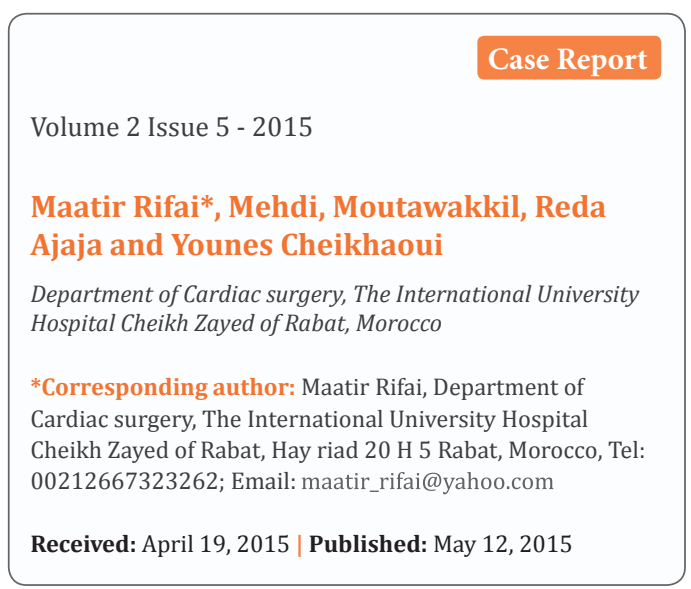

\section{Patients and Methods}

Our work is based on a retrospective study of 9 cases of irregular form of Tetralogy of Fallot with abnormal coronary arteries, collected between 1 January 2008 and 31 December 2011 at the cardiac surgery unit at the University Hospital Cheikh Zaid of Rabat. All patients underwent a complete clinical examination with chest $\mathrm{x}$-ray; echocardiography in some cases a CT angiography.

\section{Inclusion criteria}

The Inclusion criteria were abnormal VSD and abnormal right coronary artery. These anomalies were diagnosed by echocardiography, or during a catheterization. Between 2008 and 2011, among 62 patients who underwent surgical treatment for Tetralogy of Fallot, 9 patients (14.51\%) had abnormalities associated coronary arteries.

\section{Clinical data}

The mean age was 6.06 years with extreme ages ranged from 9 months to 13 years. There is a female predominance among our patients with a sex ratio of $5 \mathrm{~F} / 4 \mathrm{M}$. All patients had cyanosis, 2 patients showed severe anoxic symptoms. One patient had undergone an intervention of Blalock.

The diagnosis of coronary anomalies was determined as follows:

a) Intraoperatively in 5 patients,

b) By echocardiographic data in 2 patients

c) By angiography and catheterization in 2 patients.

The anatomical damages of coronary arteries were as follows:

a. Interventricular artery "IVA" born of the right coronary artery in 3 cases $(33.3 \%)$.

b. Right coronary artery emerging from the IVA in 2 cases (22.2\%). c. Major infundibular branch emerging from the right coronary artery in 4 cases $(44.4 \%)$.

\section{Surgical treatment}

In our series, 9 the patients underwent complete repair using cardiopulmonary bypass and intermittent cold blood cardioplegia given in an antegrade perfusion. Depending on each case, the surgical procedures of the pulmonary outflow tract enlargement were:

1) A short vertical infundibulotomy upstream and/or downstream of the abnormal coronary with enlargement by patch could be made in 6 cases $(66.6 \%)$.

2) One patient was able to achieve an expansion of the pulmonary tract by Gortex patch passed under in the right coronary artery born from the IVA and who could be mobilized in order to extend the incision below the latter and suturing the patch $(11.1 \%)$.

3) The final appearance was smooth and the right coronary artery was not stretched.

4) A trans-atrial right transpulmonary Correction was achieved in a patient (11.1\%).

5) A Contegra conduit between the right ventricle and the pulmonary artery was performed in a 16-month infant (11.1\%).

6) The pulmonary annulus was kept in 7 cases and enlarged in 2 cases.

7) The average length of clamping was 79 minutes.

8) The average duration of CPB was 139 minutes.

9) Postoperative pulmonary medium gradient average was 21 $\mathrm{mmHg}$.

10) The right ventricular pressure was significantly lower than the systemic pressures in the 9 patients who had a complete cure. 


\section{Postoperative results}

The 8 patients were extubated early and averaged at the 6 th hour of the block output. One patient required prolonged ventilatory support 72 hours. Arterial saturation in 9 patients increased from $75 \%$ on average $98 \%$. The control echocardiography objectifying a satisfactory outcome of the repair with Right ventricular pressure PVD $<50 \mathrm{mmHg}$, a mean gradient $21 \mathrm{mmHg}$ and good biventricular function. The average hospital stay was 10 days.

Medium and long term evolution: A clear improvement of respiratory functional was observed in the 9 patients. A case kept a stage NYHA II dyspnea. No respiratory or rhythmic complications were detected in our patients; the vast majority of patients improved from their states before curative surgery and currently led normal lives. These functional outcomes in the long term can be concluded that complete repair of Tetralogy of Fallot can be performed in patients with coronary artery malformation without using a conduit between the right ventricle and the pulmonary artery. The postoperative course was similar to other forms of Tetralogy of Fallot. The presence of an anomaly of the coronary artery in these patients has not resulted in increased operating risk or complication after surgical strategy adopted. We now have to analyze our long-term results whether our strategy is beneficial for these patients. The percentage of different residual lesions after complete cure was:

a. Residual VSD $22.2 \%$

b. Residual pulmonary stenosis $11.1 \%$

c. Dilatation of the right ventricle $11.1 \%$

d. Pulmonary insufficiency $11.1 \%$

e. Hypo contractility of LV none

\section{Discussion}

Congenital anomalies of the coronary arteries include a wide range of defects, and diverse gravity. Radiological explorations including imaging and interventional catheterization helped in a better surgical management. The main problem arises when there is a hypoplasia of the pulmonary annulus associated with ectopic coronary intersecting the anterior face of the infundibulum. These anomalies do not pose a problem if the pulmonary annulus is of normal size because there will be no cut on the upper part of the infundibulum. Echocardiography with different modes easily makes the diagnosis of Tetralogy of Fallot in our study. All authors confirm its reliability in the positive diagnosis of heart disease [1]. If in doubt about the origin and the path of the coronary arteries, a selective coronary angiography is performed to detect any abnormalities of the coronary arteries. In our series 2 patients underwent angiography.

\section{Surgical treatment}

The open heart surgical repair is indicated in all cases. Currently, the ideal age for elective operation is between 3 and 11 months of life. If significant symptoms from the age of 3 months, and if the anatomy is favorable, we proceed to the definitive correction rather than palliation. The medical and surgical palliation treatment allows delay the final repair and improve functional symptoms of the patient. Complete repair is performed under cardiopulmonary bypass and moderate hypothermia. It is done electively from the age of 3 months. If hypoxia is severe or if there are hypoxic crises intervention becomes essential in the newborn or young infant; it will then weigh the risks of damages against those of a palliative cure. The criteria to be considered are the experience of the surgical team, peripheral pulmonary stenosis, as well, some abnormalities of the coronary arteries.

Curative surgery includes the closure of the VSD and enlargement of pulmonary stenosis. The first vector is a short straight ventriculotomy at the infundibulum adapted to the path of the coronary artery. Some surgeons advocate a first by the right atrium and the pulmonary artery [2]. A piece of Dacron is sutured on inter ventricular communication; if significant overlap of the aorta, that part shall be bent towards the ventricle. Care is taken not to injure the bundle of His, which passes along the lower edge of the communication.

The infundubular stenosis is resected and the pulmonary valve inspected: commissurotomy is performed in cases of valvular stenosis. If the measured diameter is less than $2 \mathrm{Z}$-score, it is then necessary to cut through the pulmonary valve annulus, as well as the pulmonary trunk to enlarge it by a piece of Dacron lined pericardium (transannular patch) with or without mobilization coronary. From $20 \%$ to $40 \%$ of patients, depending on the centers, receive a transannular patch. Some surgeons prefer the establishment of a valved graft, avoiding the transannular patch: this can be justified in cases of pulmonary arteries of small caliber devices or with stenoses. Reoperation for graft replacement is obviously programmed. The procedure ends with the closing of a possible atrial septal defect. Repair using techniques based on infundibulotomy ventriculotomy or parallel to the abnormal coronary or transatrial transpulmonary way provide long-term satisfactory results [3]. The results of our study show that surgery of the irregular shape of the Tetralogy of Fallot represented by abnormalities of the coronary arteries in the early stages is beneficial in improving functional status.

\section{Results of curative surgery}

In our series, immediate postoperative residual lesions were reported in $26.3 \%$ of patients who received curative intervention. This result is close to other series where morbidity curative surgery is usually reported in $10-40 \%$ of cases [3].

\section{Conclusion}

Our work consisted of a retrospective study of 9 cases of Tetralogy of Fallot with abnormal coronary arteries collected over a period of 4 years in the cardiovascular surgery department at the International University Hospital Cheikh Zaid of Rabat. The approach most used in our study is the infundibulotomy adapted to the path of coronary with infundibular enlargement by Gortex patch, this technique allows to avoid the use of the conduit between the right ventricle and the Pulmonary artery well as palliative interventions. The postoperative result is currently excellent when the procedure is done at a young age and all children must be carefully y followed up in the long term. 


\section{References}

1. Bernard normal and pathological Y. Echocardiographie.

2. Binet JP, Hvass U, Bruniaux J, Langlois J, Planché C, et al. (1980) Complete correction of tetralogy of Fallot without opening the right ventricle. Arch Mal Coeur Vaiss 70(10): 1185-1192.

3. Kalra S, Sharma R, Choudhary SK, Airan B, Bhan A, et al. (2000) Right ventricular outflow tract after non-conduit repair of tetralogy of Fallot with coronary anomaly. Ann Thorac Surg 70(3): 723-726. 